\title{
Recombinant IgM expression in mammalian cells: A target protein challenging biotechnological production
}

\author{
Alexander Mader, Veronika Chromikova, Renate Kunert \\ Department of Biotechnology, VIBT-BOKU University of Natural Resources and Life Sciences (Vienna), Vienna, Austria \\ Email: alexander.mader@boku.ac.at
}

Received January $13^{\text {th }}, 2013$; revised March $13^{\text {th }}, 2013$; accepted April $14^{\text {th }}, 2013$

Copyright (C) 2013 Alexander Mader et al. This is an open access article distributed under the Creative Commons Attribution License, which permits unrestricted use, distribution, and reproduction in any medium, provided the original work is properly cited.

\begin{abstract}
For many years, the potential of immunoglobulin $M$ (IgM) antibodies was not fully understood because of characteristics different to the well-known immunoglobulin $G$ type like low target affinity, cross reactivity and complex protein structure. In the meanwhile IgMs have been positively evaluated for their use as therapeutic agent in the mucosal environment but also in serum to eradicate upcoming tumor cells and invading antigens. Therefore IgM class of antibodies will play a significant role in clinical applications but also diagnosis in the future. To evaluate the full potential of this kind of antibody molecules large amounts of high quality product will be needed. In this review the focus is set on the biotechnological aspect of producing IgM class antibodies recombinantly in mammalian cells. Current achievements in expression and purification of this molecule are highlighted and compared.
\end{abstract}

Keywords: IgM; Mammalian Expression; Recombinant Antibody Expression; Antibody Purification

\section{INTRODUCTION}

The immunoglobulin $\mathrm{M}$ (IgM) class of antibodies is known to be the first defense against various antigens. IgMs are produced by the immune system and play an important role in primary and adaptive humoral immune responses [1]. This class of antibodies tends to be less specific but recognizes a large variety of pathogenic antigens and has been proposed to be highly active in cytotoxic and cytolytic reactions due to superior activation of the complement system [2,3]. In the field of tumor boilogy some cancer cell markers are targeted more effectively by IgMs compared to immunoglobulin G (IgG) class antibodies [4-7] probably by higher avidity. A screening for antibodies isolated from cancer patients revealed that nearly all identified tumor specific antibodies belong to the IgM class [1].

Concerning bacterial or viral pathogens, IgMs are the first immune molecules binding membrane derived epitopes [8] indicated by Horn and coworkers who reported about an IgM against Pseudomonas aeruginosa serotype O11 which facilitates pathogen clearance by complement dependent phagocytosis [9]. Additionally the IgM class might be useful as vaccine adjuvant as it acts as soluble toll-like receptors through the formation of immune complexes with the antigen [10]. Further it has been suggested to use IgMs as preventive vaccine [11]. All these properties render IgM antibodies as attractive therapeutic agent. Despite the therapeutic benefits of IgM antibodies a major technological drawback has been identified in comparison to the classical IgG molecule. Recombinant production of this immunoglobulin class is not straight forward and has not been routinely accomplished so far. In this article we will focus on recombinant production of IgM molecules. We will highlight current achievements and shortcomings of recombinant expression in the mammalian cell system as well as purification of such molecules and discuss our findings with available literature.

\section{THE STRUCTURE OF IgM}

Like other antibody molecules the IgM class consists of heavy (HC) and light chain (LC) subunit structures that are assembled into higher polymers (Figure 1). In human serum the predominant form is the pentamer $\left(\mathrm{M}_{\mathrm{W}} \sim 950\right.$ $\mathrm{kDa})$, which is composed by five monomers connected with a polypeptide called joining-(J) chain [12,13]. Alternatively, hexamers $\left(\mathrm{M}_{\mathrm{W}} \sim 1100 \mathrm{kDa}\right)$, (six monomers without J-chain) or monomers $\left(\mathrm{M}_{\mathrm{W}} \sim 170 \mathrm{kDa}\right)$ [14] are found at rather low abundance in vivo.

The big advantage of IgMs as therapeutic agent comes from the superior mobilization of effector functions since pentameric IgMs are able to activate the complement 


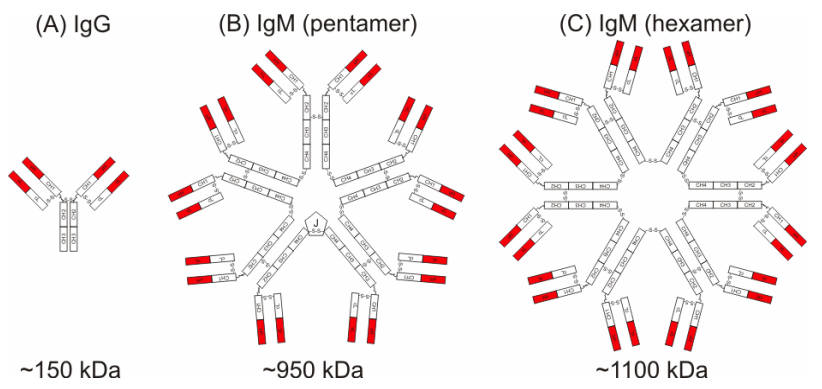

Figure 1. Structure of (A) IgG; (B) pentameric IgM; and (C) hexameric IgM.

system 100 to 1000 times better than IgG antibodies [15]. Despite monomeric IgM cannot contribute to complement activation [16] the hexameric form of IgM is even more efficient in activating complement $[2,17]$ but the strong reactivity of this form can lead to self-tissue destruction upon prolonged persistence in the human circulation system [18,19]. Additionally mucosal immunity is attained by transfer of IgM through the epithelial barrier. This passage is initiated by the J-chain of the pentameric IgM which interacts with polymeric-Ig recaptors $[20,21]$ on the epithelial membrane. Therefore, to tap the full potential of the IgM class of antibodies large amount of high quality product (mainly the pentameric form) will be needed for pre-clinical and clinical testing.

\section{RECOMBINANT EXPRESSION OF IgM}

\subsection{Antibody Productivity}

A panel of mammalian cell lines is suitable for the production of biopharmaceuticals [22] exist but the main workhorses for recombinant protein production are Chinese hamster ovary (CHO) cells. They are regarded as safe host by the US Food and Drug Administration (FDA), are able to perform efficient post-translational modifications, can be cultured in serum-free suspension in large scale bioreactors and moderate specific productivity can be overcome by gene amplification [23]. In the last decades a lot of effort has been spent on improvements in media composition and in bioprocess modifications which resulted in $\mathrm{CHO}$ fed-batch bioprocesses for antibody production that typically have specific productivities in the range of $50-60 \mathrm{pg} / \mathrm{cell} / \mathrm{day}$ and volumetric titers of $1-5 \mathrm{~g} / \mathrm{l}$ [24]. These achievements are true for IgG class molecules but for more complex antibody structures like IgM none of these values have been reported. Kunert and colleagues performed a class-switch from IgG to IgM with the potent broadly neutralizing HIV-1 antibodies 4E10 [25]. This IgM was recombinantly expressed in serum containing CHO DUKX-B11 cells resulting in cell clones with a maximum specific productivity of $10 \mathrm{pg} / \mathrm{cell} / \mathrm{day}$ compared to the IgG1 cell line which produced $15 \mathrm{pg} / \mathrm{cell} / \mathrm{day}$. In another study serum containing CHO-K1 and NS0 cells were compared for the expression of IgMs with different oligomerization status. The CHO-K1 cell line was used to obtain hexameric IgMs and the NS0 cells which constitutively expressed mouse J-chain were used to produce pentameric IgMs [26]. Both cell lines exhibited similar volumetric titers of 5 - $10 \mathrm{mg} / \mathrm{l}$. In 2009, Tchoudakova reported about a panel of IgMs that were recombinantly expressed in PER.C6 cells with specific productivities of about 20 $\mathrm{pg} / \mathrm{cell} / \mathrm{day}$ in a batch process. However, in fed batch mode only volumetric titers of $0.9-2.3 \mathrm{~g} / \mathrm{l}$ could be realized [27]. In contrast up to $8 \mathrm{~g} / \mathrm{l} \mathrm{IgG}$ in fed-batch or 25 $\mathrm{g} / \mathrm{l}$ in perfusion bioprocesses [28] were reached under serum free conditions with appreciable cell densities $\left(10^{8}\right.$ cells/ml) using PER.C6 [29].

Recently, in our group a panel of 6 different IgM molecules was expressed in CHO DUKX-B11 cells under serum free conditions in two different expression media. The MTX amplified monoclonal cell lines exhibited specific productivities in the range of $5-30 \mathrm{pg} /$ cell/day with volumetric titers of $10-140 \mathrm{mg} / \mathrm{l}$ (unpublished data).

To conclude on the reviewed cell culture data the IgM molecule turns out to be the more challenging antibody class to be expressed recombinantly compared to the IgG form. However, until to date only a few reports are published that focus on recombinant IgM expression and it seems that we are now standing at the point where we were with IgG molecules 20 years ago. Regarding the explosive developments with IgG expression over the last two decades there must be still sufficient space for further improvements for production of IgM class antibodies.

\subsection{Product Quality and Biological Function}

Besides the request to have high antibody titer producing cell lines a major focus directs to the product quality of the expressed molecules. In terms of IgM the oligomerization status of the secreted molecule is highly important for activating the effector function adequately. To utilize the full potential as complement activator the desired IgM structure is the pentamer assembly. Kunert and colleagues expressed three potent broadly neutralizing HIV-1 antibodies namely 2F5, 2G12 [30] and 4E10 [25] that have been class-switched from IgG to IgM in CHODUKX-B11 cells. The IgM type of 2G12 had a significantly increased neutralization activity but this effect was not seen for 2F5 and 4E10. However 2F5 IgM in contrast to the IgG variant was able to neutralize transepithelial HIV-1 entry in vitro which proves function of IgM class for mucosal immunity. Quality of the IgMs was controlled by 3\% - 8\% denaturing non-reducing polyacrylamide gels with the culture supernatants and a more orless pronounced fraction of monomers and in case of 
4E10 HC/LC fragments were identified additionally to the pentameric fraction. Gilmour and co-workers reported on class-switching an IgG antibody targeting blood groups Kell antigens to IgM class for use as diagnostic agent to facilitate direct agglutination of red blood cells [26]. They expressed the hexameric form of this IgM in CHO-K1 and the pentameric form in NS0 cells. They analysed the oligomerization status of the purified IgM antibodies with denaturing $10 \%$ and $12 \%$ SDS-gels under reducing conditions and showed that the CHO-K1 cell line secreted only HC and LC and the NS0 cell line additionally expressed J-chains. However in a $4 \%$ nonreducing gel mainly the pentameric band and slightly hexameric and monomeric bands were visible in both cell lines suggesting that even the CHO-K1 cell line without J-chain produced mainly the pentameric form. An interesting report about recombinant IgM expression was presented by Azuma and colleagues in 2007. They recombinantly expressed an IgM that binds to ganglioside GM3 and kills GM3 positive human myeloma cells in the presence of complement. The hexameric form without J-chain and the pentameric form were expressed in CHO-DG44 cells and compared to the parental Epstein-Barr-Virus (EBV) transformed B-cell line [31]. They analysed the purified IgMs with 3.7\% non-reducing gels and evaluated the band distribution by densitometry. The original B-cell line derived IgMs had 19.8\% hexamers, $73.6 \%$ pentamers $3.7 \%$ tetramers and $2.9 \%$ higher molecular weight bands that were defined as aggregates. In comparison the recombinant CHO-DG44 cell line derived IgM including J-chain gene had only $4.5 \%$ hexamers, $91.5 \%$ pentamers and $4 \%$ aggregates whereas the cell line without J-chain consists of $79.2 \%$ hexamers, 9.3\% pentamers, $3.5 \%$ tetramers and $6.5 \%$ aggregates. Further they could prove that the purified hexameric IgMs had a 5 - 10 fold more efficient complement dependent cytotoxicity when compared to the pentameric IgMs. However using the $3.7 \%$ gel for product analytics the lowest oligomeric form that was in the separation range was the tetramer. If these cell lines had an accompanying monomeric fraction could not be resolved using this analytic method. In 2010 Tarköy and co-workers reported a different problem of an IgM against an Opolysaccharide of $P$. aeruginosa which was produced in a hybridoma cell line [32]. After analysing freshly prepared and frozen purified IgM preparations by size exclusion chromatography and asymmetric field-flow fractionation they found two molecular weight fractions, namely the $900 \mathrm{kDa}$ pentameric IgM fraction but also an additional $1700 \mathrm{kDa}$ IgM fraction. The amount of this hypothesized dipentameric IgM fraction was $10 \%$ in the frozen batch and 3\% - 4\% in the freshly prepared batch. After separation of the di-pentameric form from the pentameric form the biological activity of both fractions was comparable. Aggregation of therapeutic molecules are problematic in course of inducing strong immunogenic reactions [33] and therefore also a relevant issue for regulatory agencies. These data suggest that product quality is influenced either by the cell type and/or the particular IgM molecule used for expression. In consistence with the literature we found different oligomerization states of individual IgM molecules that have been expressed in the same host cell line with the same expression constructs (unpublished date). In this content the importance of analytical methods for the determination of the quality of the expressed IgMs becomes evident. Most studies reviewed in this section used nonreducing $3.7 \%$ - $4 \%$ gels to determine the rate of pentamer formation. How- ever the detection range of such gels is narrow and lower molecular weight fractions are not visible. Recently, Vorauer-Uhl proposed an optimized method to analyze and quantify the IgM oligomerization status using gradient gels combined with Sypro ${ }^{\circledR}$ Ruby staining and densitometric evaluation [34]. This optimized method is capable of analyzing multimers, isoforms and fragments simultaneously in molecular range of 25 - $1200 \mathrm{kDa}$.

\section{PURIFICATION OF IgMs}

Nearly three decades ago a method to purify monoclonal IgMs via isoelectric precipitation and subsequent gel chromatography was publish [35]. With this protocol a purity of $99 \%$ and a product recovery of $40 \%$ could be achieved when purifying monoclonal IgM from hybridoma supernatant. Since then the development of optimized and more efficient methods to purify IgMs with increased product recovery have evolved. In 2007 Gagnon and coworkers presented a three-step purification strategy for IgM antibody molecules at a conference on purification of biological products [36]. They used ceramic hydroxyapatite (CHT) for a first enrichment step leading to $90 \%$ purity and 79\% recovery, followed by anion (AIX) and cation (CIX) exchange steps. After the last purification step the purity was $99 \%$ and the recovery rate was between 51\% and 80\%. Gagnon proposed in 2009 that the CHT matrix has an advantage over the classical ion exchangers (CIX and AIX) or hydrophobic interaction (HIC) and size exclusion (SEC) chromatography because a purity greater $90 \%$ can be achieved in a single step [37]. Combination of CHT and phosphate gradient elution enabled the remove of the monomeric from the pentameric IgM fraction. However higher molecular weight aggregates cannot be separated with this method. A major drawback of most of these mentioned strategies is that they focus on an individual antibody molecule for optimization of a certain purification method. Tscheliessnig and colleagues performed a two-step purificationprocedure using polyethylene glycol (PEG) precipitation 
followed by AIX with nine different monoclonal IgM molecules from hybridoma culture supernatant [38]. They reported that product recovery was dependent on the IgM molecule and ranged from $28 \%$ - $84 \%$, whereas the purity was greater $96 \%$ except for two IgMs where only $46 \%$ and $85 \%$ purity could be achieved. In this study the influence of genomic DNA (gDNA) contamination in the culture supernatant during the purification process was also described and purification process improvements could be achieved by initial gDNA digestion with benzonase. The phenomenon of DNA impurities in IgM preparations was also reported two years later by another group [39]. A significant amount of gDNA was identified to be complexed with the IgM molecules already in the culture supernatant. The amount of gDNA was calculated as $24 \%$ of the overall mass and this IgM:DNA complex elutes within the same retention volume as non complexed IgM in SEC. Recently a novel SEC method called steric size exclusion chromatography (SXC) was reported [40]. The principle of this method is that retention of the target molecule to the hydrophilic matrix is achieved by steric exclusion of water by PEG. Elution is facilitated by lowering the PEG concentration and results in $90 \%$ purity and $90 \%$ product recovery. Often the recovery rate is influenced by the product concentration in the culture supernatant. With low producing clones (around $20 \mathrm{mg} / \mathrm{l})$ a recovery of only $70 \%$ could be achieved whereas high producing clones (50 - $100 \mathrm{mg} / \mathrm{l}$ ) performed much better. In the last years novel commercially available affinity matrices for the application with human IgMs came to market. One of these novel purification tools is the CaptureSelect ${ }^{\circledR}$ IgM matrix that was presented at a conference in 2011 [41]. In this approach 14 $\mathrm{kDa}$ Llama antibody fragments that recognize the human IgM $\mu$-chain are coupled to sepharose beads. Applying our recombinantly expressed IgM molecules we experienced a more than $95 \%$ purity using this affinity matrix (unpublished data). Nevertheless the maximum product recovery rate was between $50 \%-80 \%$.

In summary, a lot of efforts have been undertaken in the last decade to improve IgM purification. Different classical purification approaches haven been combined and adapted to IgM molecules and novel strategies haven been proposed providing an increased product recovery and nearly 100\% purity (Table 1). However it seems that different IgM molecules behave differently so that adaption of the method to the particular molecule needs to be performed.

\section{CONCLUDING REMARKS}

Today an increasing number of newly identified target antibodies against a variety of diseases belong to the IgM class. Reports on biological function and potential use of this class of antibody therapeutics are steadily rising.
Table 1. Purification strategies for IgM antibody molecules.

\begin{tabular}{ccccc}
\hline & $\begin{array}{c}\text { 3-step strategy } \\
\text { CHT/AIX/CIX }\end{array}$ & $\begin{array}{c}\text { 2-step strategy } \\
\text { PEG/AIX }\end{array}$ & SXC $^{\mathrm{c}}$ & $\begin{array}{c}\text { Capture } \\
\text { Select }^{\circledR}\end{array}$ \\
\hline Purity & $99 \%$ & $96 \%$ & $90 \%$ & $95 \%$ \\
Recovery & $51 \%-80 \%$ & $28 \%-84 \%$ & $90 \%$ & $50 \%-80 \%$ \\
\hline
\end{tabular}

${ }^{\mathrm{a}}$ Ref. [36,37]; ${ }^{\mathrm{b}}$ Ref. [38]; ${ }^{\mathrm{c}} \mathrm{Ref}$. [40].

Despite this fast-paced biological field the biotechnological aspect of producing these IgM antibodies is trailing behind. Evaluation of the full potential of IgM as therapeutic proteins demands large quantities of high quality product for further studies. After reviewing the available literature it is clear that the improvements gained with IgG type molecules have not been achieved with IgMs so far. Independent of the cell lines used the volumetric product titers are approximately 10 -fold lower when compared to optimized IgG bioprocesses. Further it seems that the product quality in terms of the oligomerization state depends on the particular IgM molecule used for expression. This in turn challenges the purification specialists, who have to deal with heterogenic IgM fractions in culture supernatants dependent on the selected IgM molecule and the process conditions. In the future a focus should be set on structural and biochemical analysis of distinct IgM molecules to probably reveal the differences in expression and product quality.

In the last years IgM class antibodies have attracted biotechnologist which is demonstrated by an increasing number of publications. To date production of IgM molecules may still be more challenging than for IgG antibodies but there is still enough space for further improvements.

\section{REFERENCES}

[1] Vollmers, H.P. and Brändlein, S. (2009) Natural antibodies and cancer. New Biotechnology, 25, 294-298. doi:10.1016/j.nbt.2009.03.016

[2] Collins, C., Tsui, F.W. and Shulman, M.J. (2002) Differential activation of human and guinea pig complement by pentameric and hexameric IgM. European Journal of Immunology, 32, 1802-1810.

doi:10.1002/1521-4141(200206)32:6<1802::AID-IMMU 1802>3.0.CO;2-C

[3] Wiersma, E.J., Collins, C., Fazel, S. and Shulman, M.J. (1998) Structural and functional analysis of J chain-deficient IgM. The Journal of Immunology, 160, 5979-5989.

[4] Irie, R.F., Ollila, D.W., O’Day, S. and Morton, D.L. (2004) Phase I pilot clinical trial of human IgM monoclonal antibody to ganglioside GM3 in patients with metastatic melanoma. Cancer Immunology, Immunotherapy, 53, 110117. doi:10.1007/s00262-003-0436-1

[5] Jones, P.C., Sze, L.L., Liu, P.Y., Morton, D.L. and Irie, R.F. (1981) Prolonged survival for melanoma patients 
with elevated IgM antibody to oncofetal antigen. Journal of the National Cancer Institute, 66, 249-254.

[6] Ollert, M.W., David, K., Schmitt, C., Hauenschild, A., Bredehorst, R., Erttmann, R., et al. (1996) Normal human serum contains a natural IgM antibody cytotoxic for human neuroblastoma cells. Proceedings of the National Academy of Sciences of the United States of America, 93, 4498-4503. doi:10.1073/pnas.93.9.4498

[7] Vollmers, H.P., O’Connor, R., Müller, J., Kirchner, T. and Müller-Hermelink, H.K. (1989) SC-1, a functional human monoclonal antibody against autologous stomach carcinoma cells. Cancer Research, 49, 2471-2476.

[8] Casali, P. and Schettino, E.W. (1996) Structure and function of natural antibodies. Current Topics in Microbiology and Immunology, 210, 167-179. doi:10.1007/978-3-642-85226-8_17

[9] Horn, M.P., Zuercher, A.W., Imboden, M.A., Rudolf, M.P., Lazar, H., Wu, H., et al. (2010) Preclinical in vitro and in vivo characterization of the fully human monoclonal IgM antibody KBPA101 specific for Pseudomonas aeruginosa serotype IATS-O11. Antimicrobial Agents and Chemotherapy, 54, 2338-2344. doi:10.1128/AAC.01142-09

[10] Ilag, L.L. (2011) Immunoglobulin $M$ as a vaccine adjuvant. Medical Hypotheses, 77, 473-478. doi:10.1016/j.mehy.2011.06.013

[11] Yang, Q.E. (2009) IgM, not IgG, a key for HIV vaccine. Vaccine, 27, 1287-1288. doi:10.1016/j.vaccine.2008.12.030

[12] Frutiger, S., Hughes, G.J., Paquet, N., Lüthy, R. and Jaton, J.C. (1992) Disulfide bond assignment in human J chain and its covalent pairing with immunoglobulin M. Biochemistry, 31, 12643-12647. doi:10.1021/bi00165a014

[13] Niles, M.J., Matsuuchi, L. and Koshland, M.E. (1995) Polymer IgM assembly and secretion in lymphoid and nonlymphoid cell lines: Evidence that $\mathrm{J}$ chain is required for pentamer IgM synthesis. Proceedings of the National Academy of Sciences of the United States of America, 92, 2884-2888. doi:10.1073/pnas.92.7.2884

[14] Brewer, J.W., Randall, T.D., Parkhouse, R.M. and Corley, R.B. (1994) Mechanism and subcellular localization of secretory IgM polymer assembly. The Journal of Biological Chemistry, 269, 17338-17348.

[15] Racine, R. and Winslow, G.M. (2009) IgM in microbial infections: Taken for granted? Immunology Letters, 125, 79-85. doi:10.1016/j.imlet.2009.06.003

[16] Metzger, H. (1970) Structure and function of gamma M macroglobulins. Advances in Immunology, 12, 57-116. doi:10.1016/S0065-2776(08)60168-6

[17] Randall, T.D., King, L.B. and Corley, R.B. (1990) The biological effects of IgM hexamer formation. European Journal of Immunology, 20, 1971-1979. doi:10.1002/eji.1830200915

[18] Brewer, J.W., Randall, T.D., Parkhouse, R.M. and Corley, R.B. (1994) IgM hexamers? Immunology Today, 15, 165168. doi:10.1016/0167-5699(94)90313-1

[19] Hughey, C.T., Brewer, J.W., Colosia, A.D., Rosse, W.F. and Corley, R.B. (1998) Production of IgM hexamers by normal and autoimmune B cells: Implications for the phy- siologic role of hexameric IgM. The Journal of Immunology, 161, 4091-4097.

[20] Reddy, P.S. and Corley, R.B. (1999) The contribution of ER quality control to the biologic functions of secretory IgM. Immunology Today, 20, 582-588. doi:10.1016/S0167-5699(99)01542-X

[21] Johansen, F.E., Braathen, R. and Brandtzaeg, P. (2000) Role of $\mathrm{J}$ chain in secretory immunoglobulin formation. Scandinavian Journal of Immunology, 52, 240-248. doi:10.1046/j.1365-3083.2000.00790.x

[22] Butler, M. and Meneses-Acosta, A. (2012) Recent advances in technology supporting biopharmaceutical production from mammalian cells. Applied Microbiology and Biotechnology, 96, 885-894. doi:10.1007/s00253-012-4451-z

[23] Kim, J.Y., Kim, Y.G. and Lee, G.M. (2012) CHO cells in biotechnology for production of recombinant proteins: Current state and further potential. Applied Microbiology and Biotechnology, 93, 917-930. doi:10.1007/s00253-011-3758-5

[24] De Jesus, M. and Wurm, F.M. (2011) Manufacturing recombinant proteins in kg-ton quantities using animal cells in bioreactors. European Journal of Pharmaceutics and Biopharmaceutics, 78, 184-188. doi:10.1016/j.ejpb.2011.01.005

[25] Kunert, R., Wolbank, S., Stiegler, G., Weik, R. and Katinger, H. (2004) Characterization of molecular features, antigen-binding, and in vitro properties of IgG and IgM variants of 4E10, an anti-HIV type 1 neutralizing monoclonal antibody. AIDS Research and Human Retroviruses, 20, 755-762. doi:10.1089/0889222041524571

[26] Gilmour, J.E., Pittman, S., Nesbitt, R. and Scott, M.L. (2008) Effect of the presence or absence of $J$ chain on expression of recombinant anti-Kell immunoglobulin $\mathrm{M}$. Transfusion Medicine and Hemotherapy, 18, 167-174. doi:10.1111/j.1365-3148.2008.00853.x

[27] Tchoudakova, A., Hensel, F., Murillo, A., Eng, B., Foley, M., Smith, L., et al. (2009) High level expression of functional human IgMs in human PER.C6 cells. MAbs, 1, 163-171.

[28] Kuczewski, M., Schirmer, E., Lain, B. and Zarbis-Papastoitsis, G. (2011) A single-use purification process for the production of a monoclonal antibody produced in a PER.C6 human cell line. Biotechnology Journal, 6, 56-65. doi:10.1002/biot.201000292

[29] Havenga, M.J., Holterman, L., Melis, I., Smits, S., Kaspers, J., Heemskerk, E., et al. (2008) Serum-free transient protein production system based on adenoviral vector and PER.C6 technology: High yield and preserved bioactivity. Biotechnology and Bioengineering, 100, 273-283. doi:10.1002/bit.21757

[30] Wolbank, S., Kunert, R., Stiegler, G. and Katinger, H. (2003) Characterization of human class-switched polymeric (immunoglobulin $\mathrm{M}$ [IgM] and IgA) anti-human immunodeficiency virus type 1 antibodies 2F5 and 2G12. Journal of Virology, 77, 4095-4103. doi:10.1128/JVI.77.7.4095-4103.2003

[31] Azuma, Y., Ishikawa, Y., Kawai, S., Tsunenari, T., Tsu- 
noda, H., Igawa, T., et al. (2007) Recombinant human hexamer-dominant IgM monoclonal antibody to ganglioside GM3 for treatment of melanoma. Clinical Cancer Research, 13, 2745-2750.

doi:10.1158/1078-0432.CCR-06-2919

[32] Tarköy, M., Wyss, M. and Rudolf, M.P. (2010) A comparative characterization of dipentameric $(\operatorname{IgM})(2)$ and pentameric IgM species present in preparations of a monoclonal IgM for therapeutic use. Journal of Pharmaceutical and Biomedical Analysis, 51, 1084-1090. doi:10.1016/j.jpba.2009.11.003

[33] Harris, R.J., Shire, S.J. and Winter, C. (2004) Commercial manufacturing scale formulation and analytical characterization of therapeutic recombinant antibodies. Drug Development Research, 61, 137-154. doi:10.1002/ddr.10344

[34] Vorauer-Uhl, K., Wallner, J., Lhota, G., Katinger, H. and Kunert, R. (2010) IgM characterization directly performed in crude culture supernatants by a new simple electrophoretic method. Journal of Immunological Methods, 359, 21-27.

[35] Steindl, F., Jungbauer, A., Wenisch, E., Himmler, G. and Katinger, H. (1987) Isoelectric precipitation and gel chromatography for purification of monoclonal IgM. Enzyme and Microbial Technology, 9, 361-364.
[36] Gagnon, P., Hensel, F. and Richieri, R. (2007) Recent advances in the purification of IgM monoclonal antibodies. 3rd Wilbio Conference on Purification of Biological Products, Waltham, 24-26 September 2007. http://www.validated.com/revalbio/pdffiles/PUR07a.pdf

[37] Gagnon, P. (2009) Monoclonal antibody purification with hydroxyapatite. New Biotechnology, 25, 287-293.

[38] Tscheliessnig, A., Ong, D., Lee, J., Pan, S., Satianegara, G., Schriebl, K., et al. (2009) Engineering of a two-step purification strategy for a panel of monoclonal immunoglobulin $\mathrm{M}$ directed against undifferentiated human embryonic stem cells. Journal of Chromatography A, 1216, 7851-7864.

[39] Gagnon, P., Hensel, F., Lee, S. and Zaidi, S. (2011) Chromatographic behavior of IgM: DNA complexes. Journal of Chromatography A, 1218, 2405-2412.

[40] Lee, J., Gan, H.T., Latiff, S.M.A., Chuah, C., Lee, W.Y., Yang, Y.-S., et al. (2012) Principles and applications of steric exclusion chromatography. Journal of Chromatography $A, \mathbf{1 2 7 0}, 162-170$.

[41] Hanala, S. (2012) The new ParaDIgm: IgM from bench to clinic: November 15-16, 2011, Frankfurt, Germany. MultiAgent-Based Simulation (MABS), 4, 555-561. 